To Cite this paper:

Zhang, Q. and Min, G. (2019). 'Chinese writing composition among CFL learners: A comparison between handwriting and typewriting'. Computers and Composition, Vol 54. doi: https://doi.org/10.1016/j.compcom.2019.102522

\title{
Chinese writing composition among CFL learners: A comparison between handwriting and typewriting
}

Qi Zhang ${ }^{a *}$ and Ge Min ${ }^{b}$

${ }^{a}$ School of Applied Language and Intercultural Studies, Dublin City University, Dublin,

Ireland; ${ }^{b}$ Department of Modern Languages, Chester University, UK.

Situated in the context of CFL (Chinese as a foreign language), the current study examines and compares texts produced by twelve pre-intermediate CFL learners using both pen-and-paper and the pinyin input system. The participants were also invited for interviews to investigate their attitudes towards handwriting and typewriting. Because of the ease of use of the pinyin input system, CFL learners tend to prefer it over writing by hand when composing lengthy texts. Based on the evaluations of fifteen professional CFL teachers, the typewritten texts were rated higher than the handwritten ones. Using the self-report empathy test, there was no significant correlation between an evaluator's empathy and his/her rating for the texts, whether composed by hand or with pinyin input. Pedagogically, typewriting might better assist Chinese language learning after handwriting has been introduced and practised among non-beginner CFL learners. The empathy effect on handwriting reported in previous literature is not found in the study. The study goes beyond the factors influencing typewriting and typewritten essays, to encourage future research investigating when to introduce computer-based writing and how it would best assist in language learning.

Keywords: Chinese, writing composition, handwriting, typewriting, pinyin input

\section{Introduction}

With advances in language processing technology, writing on digital devices is an essential part of everyday life in the digital era. Because of the frequent use of electronic devices for communication nowadays, typewriting must inevitably be introduced and taught to learners of Chinese as a foreign language (henceforth CFL) in order to cope with life in contemporary society. However, the impact of technology also seems to be the main reason for the marginalisation of handwriting in our daily life and consequently a reduced 
pedagogical focus on handwriting in language classrooms. In particular, Chinese requires more attention to character composition by hand, in comparison to using input software. Chinese handwriting pairs the movement patterns, usually stroke sequencing through wellpractised writing (Parkinson, Dyson, \& Khurana, 2010), with the language stimuli, namely characters. This pairing can help establish long-lasting motor memories of Chinese characters which are exploited in the orthographic recognition process. As a result, the write-to-read effect in Chinese makes handwriting necessary in the study of Chinese language, especially among CFL learners (Guan, Liu, Chan, Ye, \& Perfetti, 2011; Q. Zhang \& Reilly, 2015).

While the current in-class practice of Chinese language learning tends to rely on handwriting, input software is the main medium of writing in real life nowadays. Even for native Chinese children, typing on a computer and touchscreen is taught during the third grade of primary school (Spitzer, 2014). In addition, some official language proficiency tests are computer-based, requiring that CFL learners use input software to type Chinese characters in order to complete the writing tests (D. Zhang, 2017). For example, the Hanyu Shuiping Kaoshi (widely known as the HSK), China's national standardised test of Chinese language proficiency, offers a computer-based mode which uses pinyin ${ }^{1}$ input (Hanban Test Centre website, 2018). The writing section of Taiwan's Test of Chinese as a Foreign Language (TOCFL; D. Zhang, 2017, p. 78) is also computer-based. Computerised input is also employed for selected sections of national Chinese exams in Singapore (MOE; see also Wong et al., 2011). In this context, the current study conducts an evaluation of texts produced in the two writing modalities - handwriting and typewriting - along with an investigation of the experience of the CFL learners who composed the texts and the CFL teachers' evaluations of their writing composition.

\footnotetext{
${ }^{1}$ Pinyin can generally be understood as a Romanised form used to represent the pronunciation of each Chinese character.
} 
The paper first discusses Chinese writing composition using pen and paper and input software. It outlines the challenges and advantages of handwriting in CFL learning, which leads to a discussion of the concerns associated with typewriting in Chinese language learning. It then introduces the previous research on the evaluation of handwritten and typewritten texts, including the empathy effect. The current study includes twenty texts produced by pre-intermediate CFL learners using both pen and paper and pinyin input systems. The CFL learners were also invited for interviews in order to investigate their attitudes towards handwriting and typewriting. Fifteen CFL teachers were then asked to evaluate these twenty texts in order to examine whether and to what extent handwritten texts would be rated differently from typewritten ones due to the empathy effect on handwriting. Pedagogical implications are discussed, together with data from the interview and survey, both for typewriting in the specific CFL context and for computer-based writing in language learning in general.

\section{Handwriting vs typewriting among CFL learners}

The study of the Chinese writing system among CFL learners involves learning the three constituents of Chinese characters: orthography (the shape or form of a character), phonology (the pronunciation) and semantics (the meaning). Figure 1 displays the interconnection between these three integral parts of each character. Unlike the reliable association between phonology and orthography in alphabetic languages, the phonologyorthography link is relatively unsystematic and so the contributing effect of reading on writing in Chinese is not as strong as that in English. Instead, character handwriting requires the unification of visual attention and sensorimotor action. It consequently contributes to the development of a visual-spatial memory which has a motor memory trace and can be additional assistance for the activation of visual information in the process of character recognition. Handwriting could therefore be a more critical component of learning to read. A 
lot of evidence has been found to suggest a write-to-read effect in Chinese among native speakers (Tan, Spinks, Eden, Perfetti, \& Siok, 2005; Tan, Xu, Chang, \& Siok, 2013) and CFL learners (Cao et al., 2013; Guan et al., 2011; Q. Zhang \& Reilly, 2015).

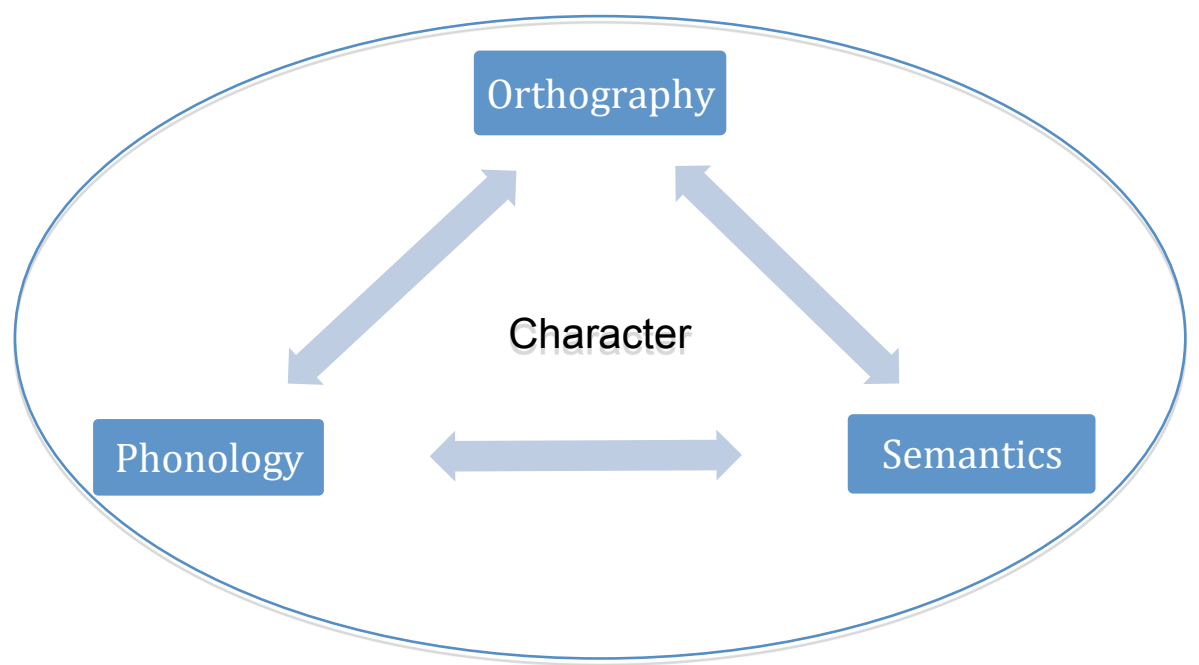

Figure 1. This shows the three constituents of a Chinese character.

Various attempts have been made to deal with the challenges associated with the study of Chinese characters, ranging from curriculum design (He \& Jiao, 2010) to different pedagogies in language classrooms (Osborne, Zhang, \& Zhang, 2018; Xu, Chang, Zhang, \& Perfetti, 2013; Q. Zhang, 2013; Q. Zhang \& Lu, 2014). One important aspect of these studies is the attempt to solve the problem of unbalanced development in reading and writing of Chinese, as well as the anxiety associated with learning Chinese characters.

The value of handwriting is undeniable in the study of the Chinese language, and it is therefore necessary to practise Chinese characters using pen and paper. However, concerns have been raised about the usefulness of handwriting in the digital era (Allen, 2008; Q. Zhang \& Lu, 2014). Learning keyboard input seems more attuned to the lifestyle of current CFL learners. It is common to use input software to type Chinese and subsequently select the intended character from a list of computer-generated possibilities. 
The current study uses the word 'typewriting' to describe the input studied. Three concepts are usually mentioned in research related to writing using a keyboard. The term 'computer-based writing' may encourage the possibility of using other functions that come with the device and the word processor software, such as online dictionaries, instant machine translation, spelling and grammar check. In contrast, 'writing with word processors' only allows writers to make use of the spelling and grammar check that comes with the word processing software. 'Typewriting', on the other hand, seems to merely suggest the writing behaviour. It is commonly understood to have the same meaning as 'writing with a word processor' in real life, since it needs to be done using word processing software. However, computer-based writing is defined in a narrow way in an exam context. The computer-based exam mentioned in section 1 only differs from conventional exams in the use of a keyboard to type rather than writing by hand (He \& Jiao, 2010). No other function is allowed to assist with writing composition. The three concepts overlap, as shown in Figure 2. The focus of the current study is on typewriting, which might be used interchangeably with 'writing with a word processor'.

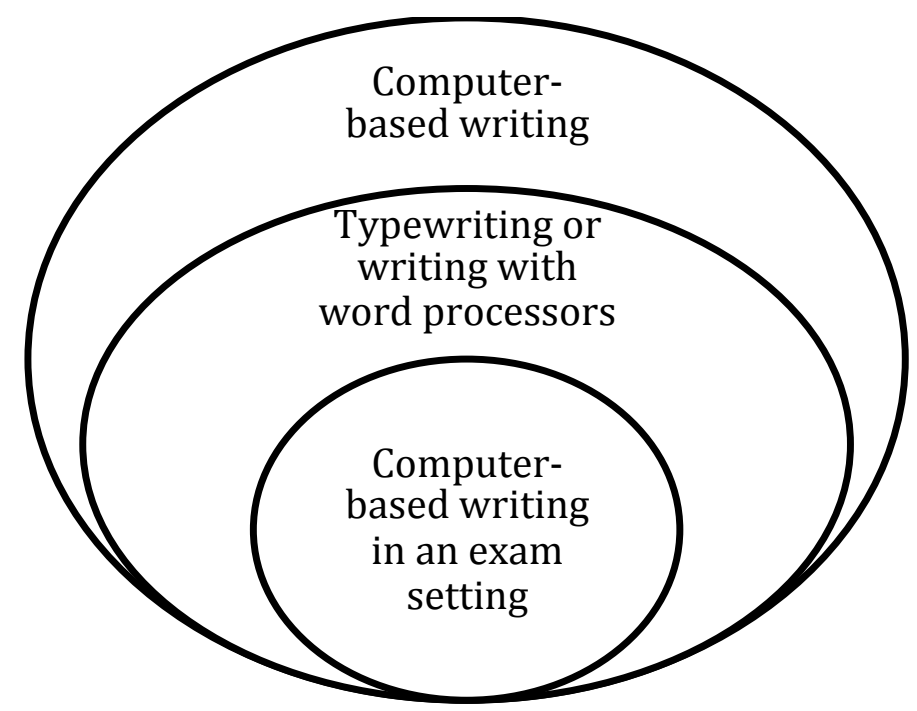

Figure 2. This is an illustration of three kinds of typewriting. 
There are two chief input methods for Chinese typewriting: component input and pinyin input, also known as stroke-based input and phonetic-based input respectively (US Patent 7,711,541 B2, 2010; US Patent No. 8,677,237 B2, 2014; US Patent No. 2005/0027534 A1, 2005). Component input allows users to draw the basic structure of a character, with the computer system then providing a list of characters with a similar structure. Pinyin input requires a user to know the pronunciation of Chinese characters. The user types the pinyin and selects the correct option from a list of characters with that pronunciation. There are a few variations of these two input methods in mainland China and other Chinese-language communities such as Taiwan and Hong Kong. However, the most common ones, such as Microsoft Pinyin, which comes with the Microsoft package, or Google Pinyin Input, which has become one of the most popular Chinese input methods, are all based on the phonetic system (see also Allen, 2008). As shown in Figure 3 below, after the writer types the pinyin (in this case, 'wu') for the character 物 (meaning 'things' or 'stuff'), the pinyin input method generates a list of characters sharing the same pronunciation. The writer then needs to be able to recognise and choose the intended character from the list, in this case the second option.

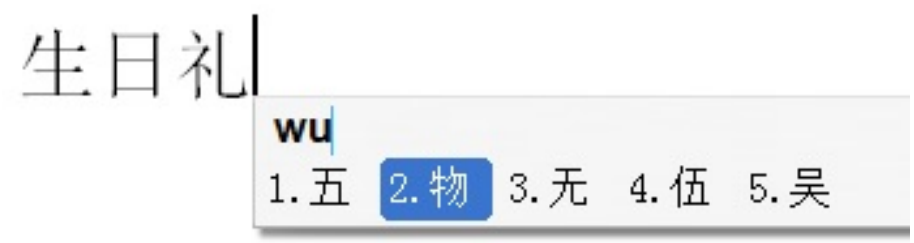

Figure 3. This is an illustration of typing with the pinyin input method.

Importantly, like other current alphabetic input methods, both component and pinyin input employ a fuzzy matching technique (Chan \& Cheung, 1992; US Patent No. 7,212,967 B2, 2007). Even if pinyin is not fully or correctly written, the pinyin input system is still able to find the most similar pronunciation according to the text input. Likewise, with component input a list of computer-generated possibilities is presented to users for selection even if a character is not structured properly by the user. As a result, the action of typewriting tends 
not to encourage memorising whole character representations. This is usually the main concern arising in previous research regarding the use of input systems for language learning (Jiang \& Zheng, 2015; Tan et al., 2013; Wang, 2012; Xing, 2008; Q. Zhang \& Reilly, 2016). Even though pinyin input seems to support the phonology-orthography link of a character in language learning, there are doubts regarding this assumption, due to the assistance from the technology. Besides, research on CFL learners shows that factors other than language proficiency play a role in the performance of typewriting Chinese, including competency level in using the pinyin input system (Wong et al., 2011), input speed (Chai, Wong, Sim, \& Deng, 2012) and amount of experience with touchscreens (Mangen, Anda, Oxborough, \& Brønnick, 2015).

Under these circumstances, the current study entailed introducing both handwriting and typewriting to a group of CFL learners in a real-life language classroom. It examines their perceptions of handwriting and typewriting in Chinese language learning, and evaluates the texts composed in two writing modalities.

\section{Typewriting Chinese with pinyin input and evaluation of typewritten texts}

Writing a Chinese character by hand is a form of construction, using the strokes to form radicals and eventually a character, which requires harmony, symmetry and equilibrium (Chang \& Yu, 2005). It indeed involves much more cognitive work and physical movement than writing with word processors. From the perspective of learners, character input systems allow them to 'write' legible and accurate characters, assisted by the fuzzy technology, in comparison with handwriting. For example, although a learner may choose the wrong character (e.g. 伍 rather than 物 in Figure 3) from a list of computer generated options, the wrongly chosen character itself (伍 in the example) is legible. This is because factors that could affect legibility - such as character height and width, number of strokes and font style have been scrutinised and controlled in order to produce legible characters on a computer 
screen (Cai, Chi, \& You, 2001; Chi, Cai, \& You, 2003). In contrast, when a character is written by hand, these factors all need to be carefully managed by the learner. Typewriting therefore requires less cognitive and physical work than handwriting. As a consequence, this may mean that CFL learners are to some extent free to focus on higher-order thinking activities. Since higher-order thinking "focus[es] on the meaning a writing intends to communicate to readers" (J. Li, 2006, pp. 7-8), typewriting can contribute to the overall quality of a text and consequently is preferred by CFL learners when producing essays (Zhu, Shum, Tse, \& Liu, 2016).

However, writing Chinese with pinyin input may not be as simple as imagined. Contrary to the two-level process experienced by L2 learners of a European language, Chinese typewriting involves a tripartite process: translating ideas from English to Chinese, transcribing Chinese characters into pinyin, and then transferring them into typing on a keyboard (Wong et al., 2011). In fact, the tripartite process consists of at least six steps for CFL learners to compose a text on a computer (ibid., p. 244):

1) Transforming ideas into words and syntactic structures in the mind

2) Recalling the pronunciation of individual characters

3) Mapping the pronunciation into pinyin

4) Mapping the pinyin representations into keyboard strokes

5) Identifying the right characters from the candidate window

6) Selecting the right characters

The complexity of composing Chinese with pinyin input is due to the lack of a phonology-to-logograph link in the Chinese writing system. Despite the fact that typewriting may help with the first step, which is related to higher-order thinking, Chai et al. (2012) point out that Chinese pinyin input, which is phonetic-based rather than alphabetic-based, may pose a greater challenge to CFL learners. 
Zhu et al. (2016) examine handwriting and typewriting from the perspective of CFL teachers, and demonstrate that typewritten Chinese texts tend to receive better evaluations than those produced with paper and pen. The ease of typing pinyin and then selecting the intended character from a list of homophones seems to improve writing quality (ibid.). On the other hand, when both characters and pinyin are handwritten, better evaluations tend to be given to writing in characters (Q. Zhang \& Reilly, 2016).

Evaluations of typewritten texts in English show a mixed picture. On the one hand, some studies demonstrate the usefulness of word processors in writing, with higher scores consequently awarded to typewritten texts among learners of English (Lam \& Pennington, 1995; J. Li, 2006; J. Li \& Cumming, 2001) and local students in the US (Russell \& Haney, 1997; Russell \& Plati, 2001). On the other hand, some studies either show that better evaluations tend to be given to handwritten texts (Breland, Lee, \& Muraki, 2005; Bridgeman \& Cooper, 1998; Chen, White, McCloskey, Soroui, \& Chun, 2011; Powers, Fowles, Farnum, \& Ramsey, 1994) or maintain that no significant difference is found between the ratings of two writing modalities (Harrington, Shermis, \& Rollins, 2000; Y.-J. Lee, 2002). Hunsu et al. (2015) point out that the key factors affecting writing outcomes are the characteristics of participants, such as language proficiency (Breland et al., 2005) and competence with word processors (Bridgeman \& Cooper, 1998; Harrington et al., 2000; Russell, 1999; Wolfe \& Manalo, 2004). However, typing skill or experience with word processors may be less of a concern to students nowadays, with their access to computers and e-devices (Hunsu, 2015).

Another factor influencing the evaluation of handwritten and typewritten texts is related to the evaluators: they may have higher expectations for texts produced with word processors, while showing more empathy for handwritten papers (Arnold et al., 1990; Powers et al., 1994; Zhu et al., 2016). Interestingly, this empathy effect is only found for handwritten Chinese characters, not for handwritten pinyin (Q. Zhang \& Reilly, 2016). In relation to 
English writing performance, although Russell and Plati (2004, p. 8) do not use the phrase 'empathy effect', they find that the evaluators "felt the handwritten essays were more personable and that they felt a stronger connection to the writer because of their handwriting". Specifically, comments such as "really tried hard" for handwritten essays demonstrate the empathy of evaluators. For the exact reason that the effect of the two kinds of presentation (handwritten and typewritten) on evaluators is unclear, as pointed out by Hunsu (2015), the current study focuses on the empathy effect mentioned in previous research. In addition, both Harrington et al. (2000) and Powers et al. (1994) advocate that a way to reduce the evaluation discrepancy is to explicitly train essay raters with extra emphasis on essay quality rather than the method of writing. Although Powers et al. (1994) suggest informing the reader of the empathy effect in order to ensure continued fairness in the evaluation, an empirical examination of whether empathy indeed plays a role in the rating of handwritten and typewritten compositions is needed. Training for raters can then be developed accordingly.

Owing to the paucity of research in typewritten text composition, only one study (Zhu et al., 2016) examines the evaluation of handwritten and typewritten texts. In this case, the current study scrutinises the evaluation of Chinese texts composed by pre-intermediate CFL learners with pen and paper and with pinyin input, in order to compare their writing performance in two writing modalities.

\section{Research design}

There were three steps. First, 12 CFL learners (aged between 18 and 23) were recruited to compose Chinese texts by hand and with pinyin input. These learners had all studied Chinese for approximately 18 months since they entered two universities - one in the UK and the other in Ireland - at which the researchers work. All participants are studying Chinese as part of their undergraduate degree. They are categorised as pre-intermediate level, 
based on more than a year's observation by their language instructors, who are also the researchers of the current study. A limit of 20 minutes was given for the learners to write on one of two topics, Christmas Gift and New Year's Gift, using one of the two writing modalities. After an interval of a week, the same learners were asked to write on the other topic, using the writing modality they did not use the previous week. Although there are a few different pinyin input methods (e.g. Google Pinyin, Sogou Pinyin), they operate on similar principles, as mentioned in section 2. The Microsoft input method embedded in the computer system had been introduced and used in the Chinese language class and consequently was adopted in the current study.

Table 1. A summary of the text compositions in two writing modalities

\begin{tabular}{llll} 
& $\begin{array}{c}\text { Christmas Gift } \\
\text { 《圣诞礼物》 }\end{array}$ & $\begin{array}{c}\text { New Year's Gift } \\
\text { 《新年礼物》 }\end{array}$ & Total \\
\hline Typewriting & 9 & 3 & 12 \\
Handwriting & 4 & 4 & $8^{\mathrm{a}}$ \\
\hline
\end{tabular}

${ }^{\mathrm{a}}$ Four missing data.

Table 1 summarises the number of texts composed in two different writing modalities under each topic. Four learners who typewrote a text did not participate in handwriting composition, and hence the total number of handwritten compositions is eight. In total, 20 texts were collected and used for the next stage of the research. At the end of the two writing compositions, these CFL learners filled in a questionnaire which collected background information including frequency of use of typewriting and handwriting.

Secondly, the CFL learners were invited for interviews in order to investigate their attitudes towards the use of handwriting and typewriting in Chinese language study. Because of the relatively small pool of participants, five semi-structured interviews were conducted with individual students, which allowed them to express their opinions in depth. The interview schedule and questions were drafted in advance and then piloted with one CFL learner. Of the five, two had encountered, in limited form, typewriting in Chinese before they 
started the course, while the other three had been introduced to typewriting when they began the Chinese programme at tertiary level.

Thirdly, the handwritten texts were scanned and stored electronically with the typewritten ones. All writing compositions were cropped and presented to fifteen evaluators in an online survey using PsyToolkit (Stoet, 2010). The survey consisted of three parts: (1) background information of the evaluators, (2) evaluations of 20 texts using a 5-point marking criteria, (3) a 40-item empathy test.

The evaluators were CFL teachers aged between 26 and 62, with five male and eleven female. The length of their CFL teaching experience varied, with an average of 9.18 years. All evaluators were based in the UK or Ireland, where the writing compositions were conducted. Therefore, their evaluations can be a good indicator of the writing performance of CFL learners in real-life classrooms.

The evaluation criteria were adapted from Zhu et al. (2016) and Nie (2009), and consisted of four sections: overall impression, theme and content, language and expression, discourse coherence (see Appendix 1). They were put on a 5-point scale, so the evaluators could choose the relevant point when filling out the survey. The final score of each text could range from 4 to 20 .

As discussed in section 3, handwritten texts tend to be rated relatively positively, possibly because of the empathy or sympathy of the evaluators. However, this issue has not been investigated from a quantitative perspective, particularly in terms of how to define it and what the empathy or sympathy refers to. Baron-Cohen and Wheelwright (2004) scrutinise the relationship between empathy and sympathy and suggest that sympathy is a subset of empathy. The instrument of measuring empathy therefore includes sympathetic elements. In other words, the empathy questionnaire adopted from Baron-Cohen and Wheelwright (ibid.) 
is general enough to be used in the context of the current study as the first attempt to explore the influence of a person's empathy on his/her evaluation of a writing composition.

There are 60 items in the Empathy Quotient, with 20 of them filler items (BaronCohen \& Wheelwright, 2004). As mentioned above, the online survey already included the evaluations of 20 texts from four perspectives: $20 \times 4=80$ questions. The 20 distractors from the Empathy Quotient were therefore removed in order to ensure the completion of the whole online questionnaire in a timely fashion. A total of 40 questions were used to measure the empathy of the evaluators, in order to avoid any fatigue. Therefore, the online survey consisted of 120 items, including 80 questions evaluating the texts and 40 items of the Empathy Quotient.

\section{Results}

The data collected from the questionnaires with writers and evaluators, CFL learners and CFL teachers respectively in the current study, were analysed in IBM SPSS (Version 24). Interview data were first transcribed and then manually coded and categorised by two researchers, each working independently. The data capturing common threads across an entire set of interviews were identified and pulled together for thematic analysis (Aronson, 1994; Mojtaba, Hannele, \& Terese, 2013). Initially, 'positive' and 'negative' attitudes were used as categories in the coding, in addition to 'advantages' and 'disadvantages'. However, a large amount of overlapping content was found when four categories were used. Besides, the attitudes were closely linked with the benefits and challenges of each writing modality. Therefore, the coding was narrowed down to two, as shown in Table 2. While the examinations are based on coherent patterns within the data set, a level of subjectivity in the process is inevitable, due to the nature of a qualitative study like this one (Joffe \& Yardley, 2004).

\subsection{CFL learner writing behaviour and perceptions of two writing modalities}


After CFL learners completed the writing compositions, a questionnaire was distributed to them regarding their daily writing behaviour. In general, none of them spent more than four hours per day practising Chinese writing. As shown in Figure 4, most spent less than two hours per day on typewriting Chinese, with the majority spending two to four hours per day on Chinese handwriting.

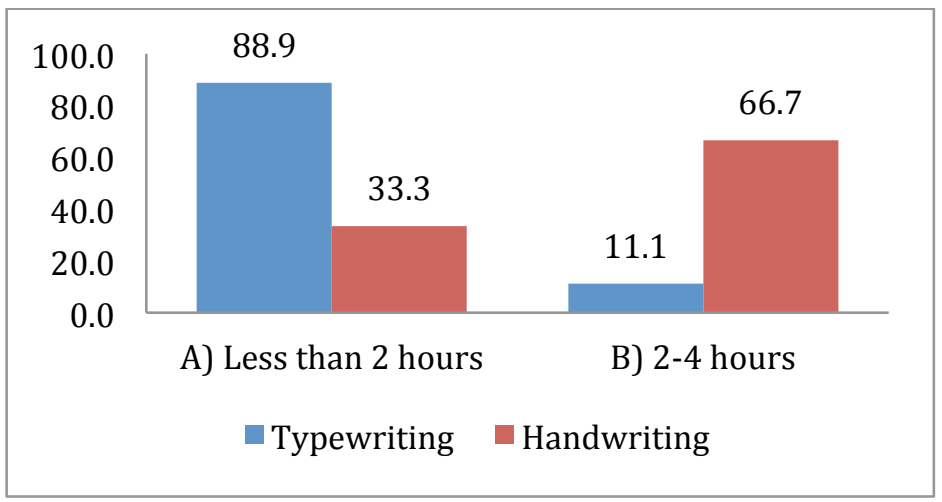

Figure 4. This shows students' average typewriting and handwriting per day as a percentage.

The interview data tease out the reasons for the heavy time investment in handwriting. All five interviewees mentioned the usefulness and effectiveness of memorising Chinese characters through handwriting. As the participants are CFL learners at pre-intermediate level, it is still an integral part of their learning process to acquire new characters and build up their vocabulary reservoir. On the other hand, interviewees pointed out that typewriting is indeed considered to be helpful in "producing short paragraphs", "using a lot of vocabulary [items]", "it is quicker to get my thoughts down", etc. This also indicates that typewriting is indeed likely to support macro-level thinking in writing compositions.

One interviewee mentioned that typewriting can help with the practice of pronunciation, since "without knowing how to say a character, you won't be able to type it". Interestingly, another interviewee pointed out that typewriting does not really help with pronunciation, since tone indicators are not used when typing, whereas tones are an essential part of Chinese listening and speaking. This shows a clear awareness of the three integral elements of a Chinese character shown in Figure 1. The employment of the pinyin input 
method may only partially contribute to establishing the link between the phonology and orthography of a character. Importantly, three interviewees stated their concerns that the reliance on pinyin input and then selecting the intended character from a list of softwaregenerated possibilities, as well as the auto-correction, does not encourage learners to engage with the language as the handwriting does, and consequently may affect their learning in the long term.

The interviews show that CFL learners seem to be very well aware of the advantages and disadvantages of handwriting and typewriting, as shown in Table 2. In other words, although no strong preference for one writing modality over the other was observed in the interviews, their general perceptions of handwriting and typewriting are likely associated with the advantages and limitations of the two modalities. The interview data echo the finding of Jiang and Zheng (2015), indicating the desire of adult CFL learners to maximise the benefits they can gain from handwriting and typewriting. Awareness of the strengths and challenges of the two writing modalities may allow them to adopt the one most suitable to the specific aspect of the Chinese language they are focusing on at any given time.

Table 2. A summary of advantages and disadvantages of handwriting and typewriting from CFL learners' perspective

\begin{tabular}{|c|c|}
\hline Handwriting & Typewriting \\
\hline \multicolumn{2}{|c|}{ Advantages } \\
\hline $\begin{array}{l}\text { - Contributes to the study of Chinese in the } \\
\text { long term } \\
\text { - Helps with memorising characters, } \\
\text { including their meanings and structures } \\
\text { - Encourages Chinese language learning, } \\
\text { including new characters and words, } \\
\text { Chinese culture, etc } \\
\text { - Improves reading proficiency }\end{array}$ & $\begin{array}{l}\text { - Reduces the anxiety of learning Chinese } \\
\text { - Helps with memorising pinyin spelling } \\
\text { - Supports higher-order thinking in writing } \\
\text { composition } \\
\text { - Helps with composing legible characters } \\
\text { and keeping writing neat and tidy even after } \\
\text { editing } \\
\text { - More efficient and faster in text composing } \\
\text { and online communication }\end{array}$ \\
\hline \multicolumn{2}{|c|}{ Disadvantages } \\
\hline - Time-consuming, inefficient & $\begin{array}{l}\text { - Does not help with the learning of } \\
\text { characters, due to reliance on pinyin input } \\
\text { and auto-correction }\end{array}$ \\
\hline
\end{tabular}


- Frustrating when learning a large quantity $\mid$ - Does not help with practising tones of characters

- Produces illegible characters or messy - Relies on characters and words already writing known

If we take a closer look at Table 2 , it is easy to see that both writing modalities can be integrated into Chinese language learning in order to compensate for the relevant limitations. For example, typewriting may ease the frustration that CFL learners experience when learning a large number of new characters; and while handwriting helps with the study of new characters and words, typewriting can contribute to consolidating existing knowledge of vocabulary and composing long texts.

The interviewees also made two recommendations on the implementation of handwriting and typewriting in Chinese language learning. First, it would be beneficial to start with handwriting. This is applicable when learning a new word from a lesson, which is a short learning period, or when beginning to learn Chinese as part of a longer learning process. Second, typewriting would help reduce anxiety and frustration, as well as equipping CFL learners with the ability to communicate in the digital world, once a fundamental knowledge of Chinese language has been established.

\subsection{Average ratings of handwritten and typewritten texts}

The 20 texts composed by CFL learners in the first stage of the project were evaluated based on scoring criteria adapted from previous research (Nie, 2009; Zhu et al., 2016). Four aspects of each text were examined: (1) overall impression, (2) theme and content, (3) language and expression, (4) discourse coherence. Each aspect contained sub-scores 1-5 and therefore the final score for each text ranged from 4 to 20 (see Appendix 1).

Fifteen evaluators who had been full-time CFL teachers were invited to evaluate the texts. The average length of their CFL teaching experience was 9.18 years when the survey 
was conducted, with minimum one year and maximum 25 years. Reliability analyses were carried out in order to examine the inter-rater reliability in their scoring.

A high degree of reliability was found between the ratings for handwritten texts (see Table 3$)$. The average ICC was .88 with $95 \%$ confidence interval from .81 to $.93(\mathrm{~F}(31$, $434)=11.57, \mathrm{p}<.001)$. Table 4 shows that a high degree of reliability was also found between the ratings for typewritten texts. The average ICC was .824 with $95 \%$ confidence interval from .73 to $.89(\mathrm{~F}(47,611)=8.01, \mathrm{p}<.001)$.

Table 3. Inter-rater reliability for scores of handwritten texts

\begin{tabular}{|c|c|c|c|c|c|c|c|}
\hline \multicolumn{8}{|c|}{ Intraclass Correlation Coefficient } \\
\hline & \multirow{2}{*}{$\begin{array}{c}\text { Intraclass } \\
\text { Correlation }^{\mathrm{b}}\end{array}$} & \multicolumn{2}{|c|}{$95 \%$ Confidence Interval } & \multicolumn{4}{|c|}{ F Test with True Value 0} \\
\hline & & Lower Bound & Upper Bound & Value & df1 & $\mathrm{df2}$ & Sig \\
\hline Single Measures & $.33^{\mathrm{a}}$ & .22 & .49 & 11.57 & 31 & 434 & .000 \\
\hline Average Measures & $.88^{\mathrm{c}}$ & .81 & .93 & 11.57 & 31 & 434 & .000 \\
\hline \multicolumn{8}{|c|}{ Note: Two-way mixed effects model where people effects are random and measures effects are fixed } \\
\hline \multicolumn{8}{|c|}{ a. The estimator is the same, whether the interaction effect is present or not. } \\
\hline \multicolumn{8}{|c|}{ b. Type A intraclass correlation coefficients using an absolute agreement definition } \\
\hline \multicolumn{8}{|c|}{ c. This estimate is computed assuming the interaction effect is absent, because it is not estimable otherwise. } \\
\hline
\end{tabular}

Table 4. Inter-rater reliability for scores of typewritten texts

\begin{tabular}{|c|c|c|c|c|c|c|c|}
\hline \multicolumn{8}{|c|}{ Intraclass Correlation Coefficient } \\
\hline & \multirow{2}{*}{$\begin{array}{c}\text { Intraclass } \\
\text { Correlation }^{\mathrm{b}}\end{array}$} & \multicolumn{2}{|c|}{ 95\% Confidence Interval } & \multicolumn{4}{|c|}{ F Test with True Value 0} \\
\hline & & Lower Bound & Upper Bound & Value & df1 & $\mathrm{df2}$ & Sig \\
\hline Single Measures & $.25^{\mathrm{a}}$ & .16 & .37 & 8.01 & 47 & 611 & .000 \\
\hline Average Measures & $.82^{\mathrm{c}}$ & .73 & .89 & 8.01 & 47 & 611 & .000 \\
\hline \multicolumn{8}{|c|}{ Note: Two-way mixed effects model where people effects are random and measures effects are fixed } \\
\hline \multicolumn{8}{|c|}{ a. The estimator is the same, whether the interaction effect is present or not. } \\
\hline \multicolumn{8}{|c|}{ b. Type A intraclass correlation coefficients using an absolute agreement definition } \\
\hline \multicolumn{8}{|c|}{ c. This estimate is computed assuming the interaction effect is absent, because it is not estimable otherwise. } \\
\hline
\end{tabular}

The inter-rater reliability was more consistent for handwritten as opposed to typewritten texts, as evidenced by a narrower confidence interval for the reliability estimate for handwritten texts (95\% confidence interval between .81 and .93$)$ than the typewritten texts (95\% confidence interval between .73 and .89$)$. In contrast, previous studies (H. K. Lee, 
2004; Zhu et al., 2016) suggest a different pattern, which is that inter-rater reliability for texts produced using a word-processor is more consistent in nature.

The table below shows the average ratings of the handwritten and typewritten texts given by 15 evaluators. A pair sample t-test was conducted. The result shows that the score of typewritten texts $(\mathrm{M}=11.12, \mathrm{SD}=1.81)$ was significantly higher than that of handwritten ones $(\mathrm{M}=12.64, \mathrm{SD}=2.19)$, with the effect size at a moderate level: $\mathrm{t}=3.833, \mathrm{df}=14, \mathrm{p}=.002,<.05$, Cohen's $\mathrm{d}=0.76, \mathrm{r}=0.36$. In other words, Chinese texts written by hand were evaluated differently from those written using the pinyin input system.

Table 5. Average scores for typewritten and handwritten texts

\begin{tabular}{|l|l|r|r|r|rr|}
\hline \multicolumn{2}{|c|}{ Paired Samples Statistics } \\
\hline & Mean & N & Std Deviation & \multicolumn{2}{|c|}{$\begin{array}{c}\text { Std Error } \\
\text { Mean }\end{array}$} \\
\hline Pair 1 & Type Score & 12.64 & & 15 & 2.19 & .56 \\
& Hand Score & 11.12 & & 15 & 1.81 & .46 \\
\hline
\end{tabular}

It is possible that other factors may have led to the variation in evaluation. As suggested by the interviewees in 5.1, CFL learners realise that they may produce illegible characters when handwriting, whereas they may also be able to write content-rich text when typewriting because of the ease of typing pinyin and selecting characters. However, it is important to note that the same participants composed the texts on two very similar topics, with an interval of only one week between handwriting and typewriting. Furthermore, all texts were randomised when presented to fifteen CFL evaluators. With all other factors as controlled as possible, the two different writing modalities are the most likely causes of this significant difference in evaluation.

\subsection{Empathy effect}

The 40 questions developed by Baron-Cohen and Wheelwright (2004) were used to measure evaluator empathy. One or two points were recorded if the answer showed mild (1 point) or strong ( 2 points) empathic behaviour. No points were recorded if the response did 
not reflect any empathic behaviour. Therefore, each person was scored 0-80 based on the empathy test.

A correlation test was conducted in order to examine whether there was an association between the evaluators' empathy and the scores they gave. As shown in Table 5, there was no significant relationship between the empathy score and the ratings for handwritten texts, $\mathrm{r}=.351, \mathrm{p}=.219$, or between the empathy and the score for typewritten texts, $\mathrm{r}=.249, \mathrm{p}=.39$.

Table 5. Correlation between empathy and scores for handwritten and typewritten texts

\begin{tabular}{|c|c|c|c|c|}
\hline \multicolumn{5}{|c|}{ Correlations } \\
\hline & & $\begin{array}{c}\text { Empathy } \\
\text { Score }\end{array}$ & Hand Score & Type Score \\
\hline Empathy Score & $\begin{array}{l}\text { Pearson Correlation } \\
\text { Sig. (2-tailed) } \\
\mathrm{N}\end{array}$ & $\begin{array}{r}1 \\
14\end{array}$ & $\begin{array}{r}.351 \\
.219 \\
14\end{array}$ & $\begin{array}{r}.249 \\
.390 \\
14\end{array}$ \\
\hline
\end{tabular}

This result is unlikely to support the previous finding that suggests empathy plays a role in the positive evaluation of texts composed by hand. It is possible that the empathy effect identified in previous research was specifically found towards writing compositions. In contrast, the empathy examined in the current study is "the drive or ability to attribute mental states to another person/animal, and entails an appropriate affective response in the observer to the other person's mental state" (Baron-Cohen \& Wheelwright, 2004, p. 168). In other words, this empathy quotient is not used to scrutinise the empathic feeling towards handwriting or typewriting.

\section{Discussion}

In general, the significantly higher score for typewritten texts seems to indicate that pre-intermediate CFL learners perform better in writing compositions assisted by pinyin input. This finding is consistent with previous research into CFL learners writing Chinese (Wong et al., 2011; Zhu et al., 2016). Handwriting Chinese requires the production of characters which process "high, nonlinear visual complexity" into a square configuration (Tan et al., 2005, p. 8781). Typewriting, on the other hand, allows CFL learners to input 
Chinese characters in perfect form. Despite the difficulties associated with typewriting with the pinyin input method, it is a less time-consuming writing modality for composing legible and well-structured characters, as also mentioned in the interviews. Therefore, instead of spending time on micro-level details (in this case, writing individual characters), writers are able to allocate their attention more towards relatively macro-level or high-order thinking, such as choosing appropriate vocabulary, structuring sentences properly, and planning the best way to present ideas in a logical order. The quality of a writing composition can consequently be improved, as demonstrated in the better evaluation of the typewritten texts compared to the handwritten ones.

As discussed in section 2, handwriting requires that writers produce the visuo-graphic properties of characters, which benefits the reading development of learners. Cao et al. (2013) find that, among a group of CFL learners, handwriting contributed positively to establish more precise orthographic representations of characters and to greater accuracy in memorising character meanings. Another study also confirms the effect of handwriting on character memorisation (Mangen et al., 2015). Although no difference between handwriting and typewriting is found in the recognition condition, performance on recalling words written by hand is significantly better than on those written using a conventional keyboard or a virtual keyboard such as on an iPad (ibid.). Similarly to handwriting characters, handwriting pinyin allows learners to reinforce a reciprocal connection between the visual representation of a pinyin word and the motor encoding of that word (Q. Zhang \& Reilly, 2015). The positive effect of handwriting is also confirmed by the participants in the current study. As a result, interviewees recommended that it would be more pedagogically beneficial to begin with handwriting and then move to typewriting.

In contrast, pinyin writing has an advantage in strengthening the phonological representations of characters and establishing the link between orthography and phonology 
(Cao et al., 2013; Jiang \& Zheng, 2015). Guan et al. (2011) further examine typewriting using pinyin input, which is "a process of associating a pointing movement on keyboards to form a character" (Q. Zhang \& Reilly, 2015, p. 348). The results also show that typewriting can support a stronger phonological representation of a character and the phonology-orthography link (Guan et al., 2011). In other words, typewriting is a potential pedagogical tool to enhance pinyin spelling and the correlation between phonology and orthography of Chinese characters.

Interestingly, the participants involved in the research of Guan et al. (2011) were CFL learners registered in the Elementary Chinese II course at Carnegie Mellon University. Based on the course description online (Carnegie Mellon University, 2018), they were not CFL beginners but were in fact similar to the participants in the current study, i.e. CFL learners at pre-intermediate level. As a result, it is reasonable to assume that typewriting may play a positive role in Chinese language learning at pre-intermediate level. This is also in line with the recommendation to begin with handwriting, as stated above. The concerns found among previous studies regarding the negative effect of using pinyin input may not be applicable to learners who have already developed a fundamental knowledge of the Chinese language. However, there would need to be a beginners vs non-beginners condition incorporated into the research design to definitively scrutinise this issue.

The current study is the first to examine the empathy effect from a quantitative perspective. Contrary to the previously claimed empathy effect on handwriting over typewriting, it does not find any significant correlation between an evaluator's empathy and his/her score for handwritten or typewritten texts. Nevertheless, it is too early to conclude that empathy does not play a role in the evaluation of handwritten or typewritten texts. As pointed out by Baron-Cohen and Wheelwright (2004), a person's empathy is sensitive to his/her emotional state and traits. The current state of a person may reduce his/her ability to switch perspectives and so may lead to lack of empathy. 
The self-report empathy measurement employed in the current study can only assess the evaluators' own beliefs about their own empathy traits. As the survey was delivered and completed online, it was impossible to monitor their state of mind when answering the empathy questions. Future research in behavioural science is needed to develop an instrument to measure empathy specifically for writing compositions, as well as to control for the emotional state of the participants.

Another interesting result contrary to Zhu et al. (2016) is the more consistent interrater reliability for handwritten as opposed to typewritten texts. The first possible explanation is that the study of Zhu et al. (ibid.) had only two raters, both with more than five years of teaching experience, meaning the inter-rater reliability test was conducted among two relatively homogeneous evaluators. In contrast, the current study involves evaluators whose teaching experience ranged between one year and 25 years. In addition, the texts from Zhu et al. (ibid.) were predominantly written by Southeast Asian CFL learners, whereas the learners in the current study are all native English-speakers living in the UK and Ireland. These factors may lead to the different results in the two studies.

Taking the more consistent inter-rater reliability for handwriting and the significantly lower rating for handwriting together, it seems that evaluators tended to be in agreement that the handwritten texts were of poorer quality than the typewritten ones. Despite the same font and font size, the same line spacing, fewer illegible characters and tidy presentation, typewritten texts were still evaluated with greater variability. A plausible explanation is that typewriting might afford more possibilities for CFL learners to demonstrate fine variations of linguistic competence. As demonstrated in the interview, learners may feel more confident about producing a lengthy paragraph. The ease of typing pinyin and selecting the intended characters, as well as the assistance of auto-correction, may allow learners to bring out as much of what they have learned as possible when writing a composition, and thus show the 
variation between learners that undoubtedly exists. This would result in greater variability in evaluations of their writing

However, this better performance is likely to happen when CFL learners already have a solid language foundation. As outlined in section 3 (Wong et al., 2011), Chinese typewriting is a tripartite process involving six steps. It would be difficult to use typewriting methods coherently without a good existing fundamental knowledge of the language, in spite of the time spent on higher-order thinking. For example, a learner needs to know the pinyin spelling of the intended character and to be able to choose the correct one from a list of homophones. The learner is unlikely to be capable of composing a text with good accuracy if he/she cannot remember the pinyin or grasp the gist of character structure.

Despite the primary focus on CFL learners' writing performance, the current study sheds light on the teaching of computer-based writing in general. First of all, the information and communication technology that penetrates almost every aspect of our daily life is also changing the characteristics of students in our classrooms. For instance, in $201384 \%$ of American households had a computer - including smartphones - with the number growing to $89 \%$ in 2016 (Ryan, 2018). Even back in 2010, almost 100\% of US university students had access to the Internet (Smith, Rainie, \& Zickuhr, 2011). A high level of Internet access and familiarity with computer usage among the young generation is evident globally. The 2012 Statistical Report on Internet Development in China shows $70.8 \%$ of all Internet users in China are aged between 10 and 39, and the majority of them are students at secondary or tertiary level (China Internet Network Information Centre, 2018). In Sweden, 90\% of students say they often or always use computers for written compositions and assignments (Nordmark, 2017). In these circumstances, with the increasing use of computers and widespread Internet availability, there is no doubt that the vast majority of students are adept computer and Internet users who are accustomed to typewriting. As a result, familiarity and 
comfort with computers may be less of a concern in terms of writing performance nowadays. Any ignorance of technology in the teaching of writing, such as typewriting in a strict sense or computer-based writing entailing computer programmes and the Internet, would exacerbate the "new digital divide between students' experience of technology outside school and their experience in the classroom" (Nordmark, 2017, p. 57). When writing with pen and paper is foreign to students, using a computer may be a better way of connecting their everyday experience with classroom practice and allowing them to present their linguistic competence with confidence.

The modern lifestyle has already made keyboard input an integral part of day-to-day communication, whereas handwriting skill has become less desirable to students. Even in the relatively extreme case of Chinese, where handwriting can have a positive impact on reading (e.g. Guan et al., 2011; Tan et al., 2013), CFL learners do not immediately abandon typewriting to favour handwriting, as shown in their interviews. Instead, choosing whether to use pen and paper or use input software seems to entail prudent planning by CFL learners in order to maximise the benefits of both writing modalities.

In other words, it is no longer a question of whether typewriting should be included in a language learning curriculum, or whether we should teach typewriting to language learners - the central question is when and how to employ computers in writing for pedagogical purposes (Hirvela, 2005; Hunsu, 2015). While introducing typewriting at pre-intermediate level might be the answer to 'when' - specifically in the CFL context - the current study also shares insight into 'how' by showing higher-order thinking to be one of the benefits of typewriting in general. As underscored by Nordmark (2017), in comparison with an emphasis on the writing products, it is vital to understand the writing process and implement processoriented writing instruction in the classroom. Consistent with previous literature (Y.-J. Lee, 2002; J. Li, 2006; Zhu et al., 2016), the current study shows that typewriting seems to assist 
writers in higher-order thinking during the writing process, contributing to the quality of writing compositions. This creates space for developing pedagogies in relation to teaching process-oriented writing. Especially for computer-based writing in a broader sense, typewriting entails other affordances of writing with technology, including online dictionaries, instant machine translation, and even real-time social networking. Since digital writing tends to be collectively and socially oriented and encourages help-seeking in the process of writing (Nordmark, 2017), pedagogical developments exploiting these technological affordances are particularly likely to support teaching the process rather than the product.

\section{Conclusion}

The current study employed a typewriting versus handwriting design to scrutinise two writing modalities through interviews with CFL learners and evaluations by CFL teachers. The overall finding suggests that typewriting seems to be preferred when higher-order thinking is required in lengthy text composition. On the other hand, typewriting does not encourage the study of Chinese characters in depth. Handwriting encourages the memorisation of characters, as indicated in the interviews, and can assist learners in character reading, as shown in previous literature, and therefore should be prioritised in the study of Chinese language. When building on a solid language foundation, CFL learners are likely to produce texts of better quality through typewriting rather than handwriting. The examination of the evaluation of the writing composition does not suggest a correlation between a rater's empathy and his/her rating of handwriting or typewriting.

Further research is needed to incorporate a beginners vs non-beginners condition in the research design, in order to scrutinise the influence of the two writing modalities on CFL learners with different levels of language proficiency. Regarding the long-discussed empathy 
effect on handwriting, an empathy measurement dedicated to examining its possible effect on writing might put the researcher in a better position to study this in depth, especially if the measurement can be carried out in controlled experimental conditions. 


\section{References}

Allen, Joseph R. (2008). Why Learning To Write Chinese Is a Waste of Time: A Modest Proposal. Foreign Language Annals, 41(2), 237-251. https://doi.org/10.1111/j.19449720.2008.tb03291.x

Arnold, Voiza, Legas, Julia, Obler, Susan, Pacheco, Mary Ann, Russell, Carolyn, \& Umbdenstock, Linda. (1990). A Study of Bias in Scoring Hand-Written vs. WordProcessed Papers. Rio Hondo College, Whittier, CA. Retrieved from https://files.eric.ed.gov/fulltext/ED345818.pdf

Aronson, Jodi. (1994). A Pragmatic View of Thematic Analysis. The Qualitative Report, 2(1), 1-3.

Baron-Cohen, Simon, \& Wheelwright, Sally. (2004). The Empathy Quotient: An Investigation of Adults with Asperger Syndrome or High Functioning Autism, and Normal Sex Differences. Journal of Autism and Developmental Disorders, 34(2), 163-175. https://doi.org/10.1023/B:JADD.0000022607.19833.00

Breland, Hunter, Lee, Yong-Won, \& Muraki, Eiji. (2005). Comparability of TOEFL CBT Essay Prompts: Response-Mode Analyses. Educational and Psychological Measurement, 65(4), 577-595. https://doi.org/10.1177/0013164404272504

Bridgeman, Brent, \& Cooper, Peter. (1998). Comparability of Scores on Word-Processed and Handwritten Essays on the Graduate Management Admissions Test. Retrieved from https://eric.ed.gov/?id=ED421528

Cai, Dengchuan, Chi, Chia-Fen, \& You, Manlai. (2001). The legibility threshold of Chinese characters in three-type styles. International Journal of Industrial Ergonomics, 27(1), 9-17. https://doi.org/10.1016/S0169-8141(00)00035-4

Cao, Fan, Rickles, Ben, Vu, Marianne, Zhu, Ziheng, Chan, Derek Ho Lung, Harris, Lindsay N., Stafura, Joseph, Xu, Yi, \& Perfetti, Charles A. (2013). Early stage visual- 
orthographic processes predict long-term retention of word form and meaning: A visual encoding training study. Journal of Neurolinguistics, 26(4), 440-461. https://doi.org/10.1016/j.jneuroling.2013.01.003

Carnegie Mellon University. (2018). Department of Modern Languages Courses in Carnegie Mellon University. Retrieved 22 April 2018, from http://coursecatalog.web.cmu.edu/dietrichcollegeofhumanitiesandsocialsciences/depar tmentofmodernlanguages/courses/

Chai, Ching Sing, Wong, Lung-Hsiang, Sim, Seok Hwa, \& Deng, Feng. (2012). Exploring the relationships between students' ability of computer-based Chinese input and other variables associated to their performances in composition writing. The Turkish Online Journal of Educational Technology, 11(3), 9.

Chan, Kwok-Ping, \& Cheung, Paul Y.S. (1992). Fuzzy-attribute graph with application to Chinese character recognition. IEEE Transactions on Systems, Man, and Cybernetics, 22(1), 153-160. https://doi.org/10.1109/21.141319

Chang, Shao-Hsia, \& Yu, Nan-Ying. (2005). Evaluation and Classification of Types of Chinese Handwriting Deficits in Elementary Schoolchildren. Perceptual and Motor Skills, 101(2), 631-647. https://doi.org/10.2466/pms.101.2.631-647

Chen, Jing, White, Sheida, McCloskey, Michael, Soroui, Jaleh, \& Chun, Young. (2011). Effects of computer versus paper administration of an adult functional writing assessment. Assessing Writing, 16(1), 49-71. https://doi.org/10.1016/j.asw.2010.11.001

Chi, Chia-Fen, Cai, Dengchuan, \& You, Manlai. (2003). Applying image descriptors to the assessment of legibility in Chinese characters. Ergonomics, 46(8), 825-841. https://doi.org/10.1080/0014013031000109214 
China Internet Network Information Centre. (2018). Statistical Report on Internet Development in China (The 41st Statistical Report on Internet Development in China). China Internet Network Information Centre. Retrieved from http://www.cnnic.cn/hlwfzyj/hlwxzbg/hlwtjbg/201808/P020180820630889299840.pd f

Guan, Connie Qun, Liu, Ying, Chan, Derek Ho Leung, Ye, Feifei, \& Perfetti, Charles A. (2011). Writing strengthens orthography and alphabetic-coding strengthens phonology in learning to read Chinese. Journal of Educational Psychology, 103(3), 509-522. https://doi.org/10.1037/a0023730

Hanban Test Centre website. (2018). Rules for the implementation of network-based HSK in test centres in China [Hanyu wangluo kaoshi guonei kaodian guanli banfa]. Retrieved 13 April 2018, from http://www.chinesetest.cn/goquestioncentent.do?id=41253562

Harrington, Susanmarie, Shermis, Mark D, \& Rollins, Angela L. (2000). The influence of word processing on English placement test results. Computers and Composition, 17(2), 197-210. https://doi.org/10.1016/S8755-4615(00)00029-3

He, Wayne W., \& Jiao, Dela. (2010). Curriculum design and special features. In Teaching and Learning Chinese: Issues and Perspectives (pp. 217-235). Charlotte, NC: Information Age Publishing.

Hirvela, Alan. (2005). Computer-based reading and writing across the curriculum: Two case studies of L2 writers. Computers and Composition, 22(3), 337-356. https://doi.org/10.1016/j.compcom.2005.05.005

Hunsu, Nathaniel J. (2015). Issues in transitioning from the traditional blue-book to computer-based writing assessment. Computers and Composition, 35, 41-51. https://doi.org/10.1016/j.compcom.2015.01.006 
Jiang, Xin, \& Zheng, Yan. (2015). A study of attitudes of learners of Chinese in China towards handwriting and keyboard inputting characters [Laihua hanyu xuexizhe hanzi shouxie he jianpan shuru taidu de diaocha yanjiu]. Research on Chinese Applied Linguistics, 1, 53-77.

Jin, Sugano. (2010). US Patent No. 7,711,541 B2. US Patent and Trademark Office.

Joffe, Helene, \& Yardley, Lucy. (2004). Content and Thematic Analysis. In Research Methods for Clinical and Health Psychology. London: Sage.

Lam, F. S., \& Pennington, Martha C. (1995). The computer vs. the pen: A comparative study of word processing in a Hong Kong secondary classroom. Computer Assisted Language Learning, 8(1), 75-92. https://doi.org/10.1080/0958822950080106

Lee, H.K. (2004). A comparative study of ESL writers' performance in a paper-based and a computer-delivered writing test. Assessing Writing, 9(1), 4-26. https://doi.org/10.1016/j.asw.2004.01.001

Lee, Young-Ju. (2002). A comparison of composing processes and written products in timedessay tests across paper-and-pencil and computer modes. Assessing Writing, 8(2), 135-157. https://doi.org/10.1016/S1075-2935(03)00003-5

Li, Dong, \& Li, Johnson. (2014). US Patent No. 8,677,237 B2. United States. Retrieved from https://patentimages.storage.googleapis.com/44/6a/7e/c2f190a03c4344/US8677237.p df

Li, Jiang. (2006). The mediation of technology in ESL writing and its implications for writing assessment. Assessing Writing, 11(1), 5-21. https://doi.org/10.1016/j.asw.2005.09.001

Li, Jiang, \& Cumming, Alister. (2001). Word Processing and Second Language Writing: a Longitudinal Case Study, 1(2), 127-152. 
Mangen, Anne, Anda, Liss G., Oxborough, Gunn H., \& Brønnick, Kolbjørn. (2015). Handwriting versus keyboard writing: Effect on word recall. Journal of Writing Research, 7(2), 227-247. https://doi.org/10.17239/jowr-2015.07.02.1

Meurs, Pim van, \& Zhang, Lu. (2005). US Patent No. 2005/0027534 A1. US Patent and Trademark Office. Retrieved from https://patentimages.storage.googleapis.com/d2/e3/88/c6d484e5d7c39c/US20050027 534A1.pdf

Mojtaba, Vaismoradi, Hannele, Turunen, \& Terese, Bondas. (2013). Content analysis and thematic analysis: Implications for conducting a qualitative descriptive study. Nursing \& Health Sciences, 15(3), 398-405. https://doi.org/10.1111/nhs.12048

Nie, Dan. (2009). A historical account of the assessment criteria for HSK writing [Hanyu shuiping kaoshi (HSK) xiezuo pingfen biaozhun fazhan gaishu]. Journal of Yunnan Normal University (Teaching and Research on Chinese as a Foreign Language), 7(6), $15-20$.

Nordmark, Marie. (2017). Writing Roles: A Model for Understanding Students' Digital Writing and the Positions That They Adopt as Writers. Computers and Composition, 46, 56-71. https://doi.org/10.1016/j.compcom.2017.09.003

Osborne, Caitríona, Zhang, Qi, \& Zhang, George Xinsheng. (2018). Which is more effective in introducing Chinese characters? An investigative study of four methods used to teach CFL beginners. The Language Learning Journal, 1-17. https://doi.org/10.1080/09571736.2017.1393838

Parkinson, Jim, Dyson, Benjamin J., \& Khurana, Beena. (2010). Line by line: the ERP correlates of stroke order priming in letters. Experimental Brain Research, 201(3), 575-586. https://doi.org/10.1007/s00221-009-2077-x 
Powers, Donald E., Fowles, Mary E., Farnum, Marisa, \& Ramsey, Paul. (1994). Will They Think Less of My Handwritten Essay If Others Word Process Theirs? Effects on Essay Scores of Intermingling Handwritten and Word-Processed Essays. Journal of Educational Measurement, 31(3), 220-233.

Russell, Michael. (1999). Testing On Computers: A Follow-up Study Comparing Performance On Computer and On Paper. Educational Policy Analysis Archives, $7(20), 1-47$.

Russell, Michael, \& Haney, Walt. (1997). Testing Writing on Computers: An Experiment Comparing Student Performance on Tests Conducted via Computer and via Paperand-Pencil. Education Policy Analysis Archives, 5(3). Retrieved from https://www.learntechlib.org/p/85891/

Russell, Michael, \& Plati, Tom. (2001). Effects of Computer Versus Paper Administrations of a State-Mandated Writing Assessment. Teachers College Record, 1-34.

Russell, Michael, \& Plati, Tom. (2004). Effects of handwriting and computer-print on composition scores- a follow-up to Powers, Fowles, Farnum,\& Ramsey. Practical Assessment, Research \& Evaluation, 9(1), 1-9.

Ryan, Camille. (2018). Computer and Internet Use in the United States: 2016 (No. CB 18TPS.40) (p. 14). United States Census Bureau. Retrieved from https://www.census.gov/content/dam/Census/library/publications/2018/acs/ACS39.pdf

Smith, Aaron, Rainie, Lee, \& Zickuhr, Kathryn. (2011). College students and technology Pew Research Center (Pew Internet \& American Life Project). U.S. Pew Research Centre. Retrieved from http://www.pewinternet.org/2011/07/19/college-students-andtechnology/ 
Spitzer, Manfred. (2014). Information technology in education: Risks and side effects. Trends in Neuroscience and Education, 3(3-4), 81-85.

https://doi.org/10.1016/j.tine.2014.09.002

Stoet, Gijsbert. (2010). PsyToolkit: A software package for programming psychological experiments using Linux. Behavior Research Methods, 42(4), 1096-1104. https://doi.org/10.3758/BRM.42.4.1096

Tan, Li Hai, Spinks, John A., Eden, Guinevere F., Perfetti, Charles A., \& Siok, Wai Ting. (2005). Reading depends on writing, in Chinese. Proceedings of the National Academy of Sciences, 102(24), 8781-8785. https://doi.org/10.1073/pnas.0503523102

Tan, Li Hai, Xu, Min, Chang, Chun Qi, \& Siok, Wai Ting. (2013). China’s language input system in the digital age affects children's reading development. Proceedings of the National Academy of Sciences, 110(3), 1119-1123. https://doi.org/10.1073/pnas.1213586110

Wang, Zulei. (2012). Writing or Inputting - Discussion on overseas students' study of Chinese character input ['Shuxie' haishi 'Shuru'? Liuxuesheng hanzi shuru xuexi zouyi]. The Journal of Modernisation of Chinese Language Education, 1(1), 71-77.

Wen, Say Ling, Chang, Zechary, \& Wu, Leger. (2007). US Patent No. 7,212,967 B2. United States Patent: US Patent and Trademark Office. Retrieved from https://patentimages.storage.googleapis.com/6c/fd/e2/a3760609aa789c/US7212967.p $\mathrm{df}$

Wolfe, Edward W, \& Manalo, Jonathan R. (2004). Composition Medium Comparability in a Direct Writing Assessment of Non-Native English Speakers. Language Learning, $8(1), 53-65$. 
Wong, Lung-Hsiang, Chai, Ching-Sing, \& Gao, Ping. (2011). The Chinese input challenges for Chinese as second language learners in computer-mediated writing: An exploratory study. The Turkish Online Journal of Educational Technology, 10(3), 17.

Xing, Xishen. (2008). A discussion of the effect of Chinese character typewriting on character recognition [Lun hanzi shuru ruhe youxiao cujin shizi jiaoxue]. E-Education Research [Dianhua Jiaoyu Yanjiu], (7), 28-30.

Xu, Yi, Chang, Li-Yun, Zhang, Juan, \& Perfetti, Charles A. (2013). Reading, Writing, and Animation in Character Learning in Chinese as a Foreign Language. Foreign Language Annals, 46(3), 423-444. https://doi.org/10.1111/flan.12040

Zhang, Dongbo. (2017). Developments in Research on Testing Chinese as a Second Language. In Chinese as a Second Language Assessment (pp. 67-87). Springer, Singapore. https://doi.org/10.1007/978-981-10-4089-4_4

Zhang, Qi. (2013). The Impact of Film and Film-based Activities on the Attitudes of Englishspeaking Secondary-school Students towards L2 Chinese, $1(2), 17$.

Zhang, Qi, \& Lu, Zhouxiang. (2014). The writing of Chinese characters by CFL learners: Can writing on Facebook and using machine translation help? Language Learning in Higher Education, 4(2). https://doi.org/10.1515/cercles-2014-0023

Zhang, Qi, \& Reilly, Ronan. (2016). The reading of handwriting: An evaluation of Chinese written by CFL learners. Presented at the Twenty-Third Annual Meeting of Society for Scientific Studies of Reading (SSSR), University of Porto, Portugal.

Zhang, Qi, \& Reilly, Ronan G. (2015). Writing to read: The case of Chinese. 29th Pacific Asia Conference on Language, Information and Computation, 345-354.

Zhu, Yu, Shum, Shiu-Kee Mark, Tse, Shek-Kam Brian, \& Liu, Jinghui Jack. (2016). Wordprocessor or pencil-and-paper? A comparison of students' writing in Chinese as a 
foreign language. Computer Assisted Language Learning, 29(3), 596-617.

https://doi.org/10.1080/09588221.2014.1000932 